\title{
Indications for lung transplant referral and listing
}

\author{
Omar Shweish, Goutham Dronavalli \\ Division of Pulmonary, Critical Care and Sleep Medicine, Baylor College of Medicine, Houston, TX, USA \\ Contributions: (I) Conception and design: All authors; (II) Administrative support: All authors; (III) Provision of study materials or patients: All \\ authors; (IV) Collection and assembly of data: All authors; (V) Data analysis and interpretation: All authors; (VI) Manuscript writing: All authors; (VII) \\ Final approval of manuscript: All authors. \\ Correspondence to: Goutham Dronavalli, MD. 6620 Main St., Ste 11B.05, Houston, TX 77030, USA. Email: dronaval@bcm.edu.
}

\begin{abstract}
Lung transplantation is a valuable therapeutic option for many patients with severe lung disease who have exhausted other medical or surgical therapies. However, since lungs are not a manufacturable organ like artificial heart valves or left ventricular assist devices, and since they are a limited resource compared to number of patients requiring the organs, the Department of Health and Human Services set the Final Rule of organ allocation in 1998. This led to development and implementation of Lung Allocation Score (LAS) in 2005. The score broadly divides lung diseases into 4 diagnostic criteria with a coefficient factor given to each category. The score is based on the prognostic factors of each patient to determine the risk of mortality without a transplant combined with the probability of patient survival post-transplant. Most of the guidelines for "Indications for referral and listing in lung transplant" is based on consensus opinion as there is limited amount of robust data and trials about this topic. The International Society for Heart and Lung Transplant (ISHLT) has published three editions for candidate selection and listing. In this article, we have attempted to highlight the guidelines and incorporated other disease specific prognostic factors that are not captured in the LAS. Ultimately, there are other factors like geographic location, height, blood group, preformed antibodies, transplant center experience, past wait times and transplant rate, availability of organs, etc., which also play a role especially when considering listing a patient for lung transplant. We also highlighted a representative disease in each category and most criteria for that disease will apply to other diseases in that category. Finally, this article does not delve into the history and reasoning behind each guideline but is meant to provide a general overview of indications and contraindications applicable in the field of adult lung transplantation.
\end{abstract}

Keywords: Lung transplantation; candidate selection; lung transplant guidelines

Submitted Feb 14, 2019. Accepted for publication May 02, 2019.

doi: $10.21037 /$ jtd.2019.05.09

View this article at: http://dx.doi.org/10.21037/jtd.2019.05.09

\section{Introduction}

Since the introduction of successful lung transplant in the early 1980's, the field of lung transplantation has seen considerable improvement in terms of candidate selection, management and outcomes. The selection process of appropriate candidates is a meticulous one. Identifying the patients who will have a clear survival benefit is one of the challenging aspects of lung transplant. Prior to 2005, lungs were allocated based solely on time on waiting list. This was unfavorable to patients with more rapidly progressing disease like idiopathic pulmonary fibrosis (IPF) who had higher mortality while waiting for transplant. The Department of Health and Human Services published the "Final Rule" In 1998 which directed the Organ Procurement Transplant Network (OPTN) to formulate policies to ensure (I) broader sharing of organs, (II) reducing the use of waiting time as an allocation criterion, and (III) equitable organ allocation using objective medical criteria and medical urgency for allocation (1). Because of the Final Rule, the Lung Allocation Score (LAS) was created and implemented in 2005. The International Society for 
Heart and Lung Transplantation (ISHLT) has published several consensus statements/guidelines on the selection and listing criteria for lung transplant in 1998, 2006 and 2015 (2-4). Despite the growing body of scientific evidence, these statements remain largely based on expert opinion, and are subject to change in light of emerging evidence. The LAS serves to prioritize lung transplant candidates based on waitlist mortality and post-transplant survival by creating a model, which is supposed to reflect a net gained post-transplant survival. In the LAS, lung disease diagnoses are divided into four main categories: (A) obstructive lung disease (chronic obstructive pulmonary disease or COPD); (B) pulmonary vascular disease (idiopathic pulmonary arterial hypertension or IPAH); (C) infectious lung disease [cystic fibrosis (CF)]; and (D) restrictive lung diseases (IPF). We herein review the most common indications for lung transplant referral and listing in each of these categories, represented by one disease for each category. We will also discuss other factors in the prognostication of some diseases and their potential impact on prioritization of lung transplant recipients.

\section{When to consider lung transplant}

Generally, lung transplant is considered as a last resort treatment modality for patients with progressive lung disease who have exhausted medical and surgical treatment. The ISHLT specifies that patients with chronic end stage lung conditions who have a high ( $>50 \%) 2$-year mortality risk without a lung transplant, along with a high likelihood $(>80 \%)$ of short-term and long-term survival provided adequate graft survival is present, are acceptable candidates for consideration of lung transplant (2). It is important to note that referral and listing for lung transplant are two different entities. The ISHLT recommends early referral to a transplant center for progressive lung diseases that have a projected poor prognosis. Referral means that a patient has met the minimal clinical criteria and further consideration towards lung transplant should be considered in the absence of any absolute contraindications. Listing, on the other hand, requires a thorough evaluation and careful risk-tobenefit assessment. In general, listing a patient for lung transplant is thought to be an explicit acknowledgment that a patient has limited life expectancy without lung transplant and the odds of survival are better with lung transplant. There are controversies regarding whether the previous statement holds true in all circumstances, especially in timing of listing for diseases like COPD wherein lung transplantation may provide an improved quality of life but not necessarily a longevity benefit for everyone.

\section{How are lungs allocated? The LAS}

Prior to the development of the LAS, donor lungs were allocated based on $\mathrm{ABO}$ match and the time accumulated on the transplant waitlist. This practice was associated with high waitlist mortality, particularly in subgroups like IPF (5). The initial lung allocation criteria remained largely unchanged until the late 1990's when the "Final Rule" was published by the US. Department of Health and Human Services (6). The goal of "The Final Rule" was to reduce waitlist mortality by emphasizing on broader sharing of organs and abandoning the use of waiting time as the sole allocation criterion. Instead, there was a trend toward the utilization of objective medical data and prioritization of candidates based on medical urgency. This ultimately led to the development of the LAS, which was first implemented in May 2005 and is still in use today. The goal of the LAS is to offer transplant to patients with higher risk of waitlist mortality while ensuring a clear survival benefit after transplant. It aims to achieve that by incorporating diagnostic and prognostic factors into a predictive module of waitlist mortality and weighs it against projected posttransplant survival (Table 1). For example, if two patients have a similar calculated waitlist mortality, the LAS is designed in a way that will prioritize lung allocation to the patient with the highest predicted post-transplant survival. The LAS was designed to be evaluated and upgraded periodically, and few additions were implemented since its development (7). The use of LAS had a significant impact on lung transplantation practices-particularly in reducing waitlist mortality, increasing number of transplants performed, and favoring patients with a higher waitlist mortality (8).

\section{Transplant statistics}

Over the last 3 decades, there has been a significant increase in the number of lung transplants performed, with the main trend seen in adult bilateral lung transplant. A recent report by the registry of ISHLT listed all lung transplants performed between 1995 and 2015. The most common indications consisted of COPD (36.5\%), interstitial lung disease (ILD) (29.7\%), and bronchiectasis (18.5\%) (9). Of these three broad categories, COPD without alpha-1 antitrypsin deficiency, idiopathic interstitial pneumonia 
Table 1 Factors included in the Lung Allocation Score

Age
BMI
Lung diagnosis group
Functional status
Presence of DM
Assisted ventilation
Supplemental oxygen
Predicted FVC percentage
6MWD
Pulmonary artery pressure, systolic
mPAP
CVP
Cl
PCO
Serum creatinine
Total bilirubin

BMI, body mass index; DM, diabetes mellitus; FVC, forced vital capacity; 6MWD, six-minute walk distance; mPAP, mean pulmonary arterial hypertension; CVP, central venous pressure; $\mathrm{Cl}$, cardiac index; $\mathrm{PCO}_{2}$, partial pressure of carbon dioxide.

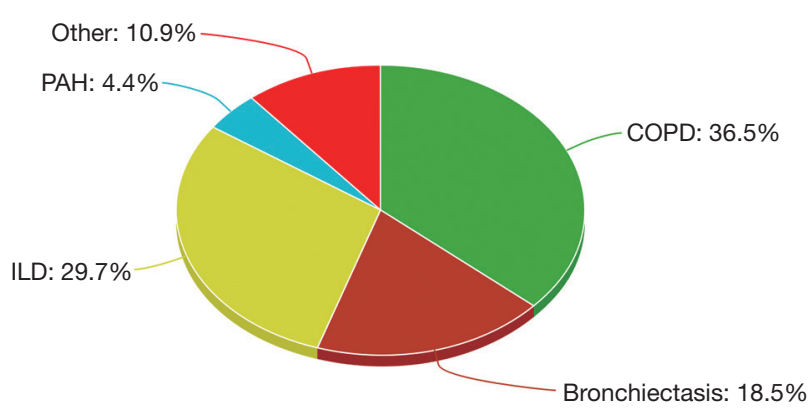

Figure 1 Primary indications for adult lung transplant between January 1995 and June 2015. COPD, chronic obstructive pulmonary disease; ILD, interstitial lung disease; $\mathrm{PAH}$, pulmonary arterial hypertension.

(IIP), and CF comprised the bulk of lung transplant, respectively (Figure 1).

\section{Contraindications to lung transplant}

Given the high risk and complexity of lung transplant,
Table 2 Contraindications to lung transplant

\author{
Absolute \\ Any untreatable malignancy, end-organ dysfunction, active \\ infection, bleeding diathesis or significant coronary artery \\ disease not amenable to revascularization \\ $\mathrm{BMI} \geq 35 \mathrm{~kg} / \mathrm{m}^{2}$ \\ Non-adherence to therapy \\ Substance abuse \\ Poor social support \\ Relative \\ General: age $>65$, class I obesity (BMI $30-34.9 \mathrm{~kg} / \mathrm{m}^{2}$ ), severe \\ malnutrition \\ Medical: Severe osteoporosis, prolonged IMV, ECLS, HIV, \\ hepatitis $\mathrm{B}, \mathrm{C}$ or infection with any highly virulent organism \\ Surgical: Pleurodesis or prior cardiothoracic surgery \\ BMI, body mass index; IMV, invasive mechanical ventilation; \\ ECLS, Extracorporeal Life Support system.
}

careful assessment of potential contraindications should be made. The most recent ISHLT guidelines cites several absolute contraindications for lung transplant (2). These generally encompass conditions that are either untreatable or are associated with higher mortality. However, different centers vary in their definition of what constitutes an absolute contraindication. Generally, the presence a medical condition that is not amenable to treatment, including malignancy, organ dysfunction, coronary artery disease, active mycobacterium tuberculosis infection, or bleeding diathesis are considered absolute contraindications. Other absolute contraindications include obesity [body mass index $\left.(\mathrm{BMI}) \geq 35 \mathrm{~kg} / \mathrm{m}^{2}\right]$, and psychosocial factors resulting in persistent non-adherence to medical therapy, medical care, poor social support, or substance abuse (see Table 2).

\section{Relative contraindications}

The ISHLT also identified several relative contraindications for lung transplant, many that are potentially reversible and thus require individual assessment (2). These can be categorized into general, medical, and surgical.

\section{General}

These include:

(I) Age $>65$ years;

(II) Class I obesity (BMI 30-34.9 kg/m²); 
(III) Severe malnutrition.

Most centers do transplant patients up to the age of 70 and some even above 70 years of age. These patients are usually in acceptable functional status and have minimal medical and surgical comorbidities or contraindications. BMI $>35 \mathrm{~kg} / \mathrm{m}^{2}$ (particularly truncal obesity) has been to show be associated with nearly 2 -fold increased mortality (10). Obesity and underweight (BMI $<18 \mathrm{~kg} / \mathrm{m}^{2}$ ) are associated with an increased risk of death after lung transplantation. More recent data have not found class I obesity to be a significant risk factor for higher mortality but most centers recommend potential candidates to strive to achieve a goal BMI $<30 \mathrm{~kg} / \mathrm{m}^{2}$. BMI is widely used to measure the burden of adiposity since it is a cheap and readily available tool, but a more comprehensive method to quantify both adiposity and sarcopenia would better serve in identifying patients at risk for worse perioperative outcomes.

\section{Medical}

These include:

(I) Severe symptomatic osteoporosis;

(II) Prolonged mechanical ventilation;

(III) Extracorporeal Life Support system (ECLS);

(IV) Infection with highly virulent and resistant organisms;

(V) HIV, hepatitis B, hepatitis C;

(VI) Medical comorbidities that are poorly controlled or expected to significantly get worse and result in end stage organ damage (diabetes mellitus, hypertension, coronary artery disease, etc.).

The presence of severe osteoporosis is usually detected by DEXA scan. Corresponding symptoms are related to multiple vertebral or hip fractures and the presence of the fractures prior to transplant are troublesome as they can be very disabling. Osteoporosis is usually expected to get worse after lung transplant due to immune suppressive medications. Carefully selected candidates on mechanical ventilation, ECLS can be successfully taken to transplant with reasonable expected outcomes. Such candidates are younger with good potential for rehabilitation and the absence of multiple organ dysfunction. Similarly, patients with HIV, hepatitis B and hepatitis C infections who have no detectable viral load, end organ damage and otherwise meet criteria for transplant can be candidates for transplant.

\section{Surgical}

Surgical contraindications mainly include pleurodesis or prior cardiothoracic surgery.

Prior cardiothoracic surgery is not a contraindication to lung transplantation. However, it does pose an increased risk of perioperative bleeding, increased need for transfusions and possible renal dysfunction. In many patients, video-assisted thoracoscopic surgery (VATS) and biopsy, chest tube insertion without pleurodesis are not significant contraindications. Extensive prior thoracic surgery, pleurodesis (chemical or surgical) and any prior radiation treatment causing pulmonary fibrosis could pose significant risk of perioperative complications (11). In wellselected patients, if the perioperative complications are minimal, there are no significant differences in medium and long-term outcomes. The combination of older age, pulmonary hypertension and prior pleural procedures pose a higher risk of perioperative complications and should be taken into account prior to selection of the candidate. The patients with pneumothorax who may be a future transplant candidate should be managed without significant difference from the usual care. Routine chest tube insertion and conservative management usually does not affect future acceptance for transplant.

\section{Disease specific indications for referral and listing}

\section{Group A: obstructive lung diseases}

The diseases included in this group A (1):

* COPD;

* Alpha-1 antitrypsin deficiency;

- Lymphangioleiomyomatosis (LAM);

* Sarcoidosis with mean pulmonary artery pressure $\leq 30 \mathrm{mmHg}$;

* Bronchiectasis including primary ciliary dyskinesia.

\section{COPD}

COPD is the most common indication for lung transplant worldwide, accounting for more than one third of all lung transplants between 1995 and 2013 (9). Several factors play into the decision to refer COPD patients for lung transplant, the most important of which are indicators of worsening functional status and spirometry. The indications for lung transplant referral and listing in COPD patients are summarized in Table 3.

Compared to other chronic lung diseases, COPD poses a unique challenge for lung transplant. The goal in every candidate is to identify a time in the course of the disease 
Table 3 Indications for referral and listing for COPD

Referral
Progressive disease despite optimal medical treatment
Not a candidate for LVRS
BODE 5-6
$\mathrm{PaCO}_{2}>50$ or $\mathrm{PaO}_{2}<60$
$\mathrm{FEV}_{1}<25 \%$ predicted
Listing
BODE $\geq 7$
FEV ${ }_{1}<15-20 \%$ predicted
$\geq 3$ exacerbations during the preceding year
1 severe exacerbation with acute hypercapnic respiratory failure
Moderate to severe PH
COPD, chronic obstructive pulmonary disease; LVRS, Lung
Volume Reduction Surgery; BODE, body mass index, airflow
obstruction, dyspnea and exercise capacity index; PaCO
partial pressure of carbon dioxide; FEV
volume in 1 second; $\mathrm{PH}$, pulmonary hypertension.

Table 4 Mortality risk factors in COPD

Hypocapnia

Older age

Lower hemoglobin

Increasing residual volume

Lower zone predominant emphysema

Lower total lung capacity

Left heart failure

COPD, chronic obstructive pulmonary disease.

when the patient is most likely to have a net survival benefit from lung transplant. This proves to be more challenging in COPD for several reasons. First, due to the chronicity and protracted course of COPD, patients may tend to live beyond the median post-transplant survival. Second, the LAS is designed to identify and prioritize patients with shorter survival. This means that COPD patients listed for lung transplant will end up having lower LAS and longer waitlist times. This, in turn, will lead to disease progression, physical deconditioning and ultimately, higher risk for worse post-transplant outcomes. This is probably accountable for the drop of the percentage of lung transplant in COPD in the period between 1999 and 2014 (9). This highlights the need to consider other mortality predictors for COPD in the evaluation of patients who are being evaluated for lung transplant (Table 4). Traditionally, the degree of airflow obstruction, measured by the forced expiratory volume in one second $\left(\mathrm{FEV}_{1}\right)$ has been used as the main predictor of mortality (12). This practice has been challenged later on by several studies that do not show a strong correlation (13). Moreover, emerging evidence has shown other parameters associated with increased mortality in this patient population. Other factors that portend a worse prognosis in COPD include frequency and severity of exacerbations (14), residual volume (RV), predominant emphysema in lower lung zones, and hypocapnia (15). In addition, several cardiopulmonary exercise testing (CPET) parameters like lower maximal workload, peak oxygen uptake, sympathetic over activity, and progressive respiratory acidosis at low intensity exercise have been identified as strong predictors of mortality as well (15-17). These factors are currently not included in LAS or the usual indications for lung transplant referral or listing. In addition, the severity and frequency of exacerbations independent of severity of disease based on BMI, airflow obstruction, dyspnea and exercise capacity (BODE) index or $\mathrm{FEV}_{1}$ portends an increased mortality risk $(14,18)$. Furthermore, each episode of acute hypercapnic respiratory failure has a high inpatient mortality and this risk is persistent in the next few years after the exacerbation (19).

Patients who meet criteria for lung volume reduction surgery (LVRS) can be referred to surgery and/or lung transplant at the same time. LVRS has been associated with improved exercise capacity without a clear survival benefit, except for patients with predominantly upper lobe emphysema and low baseline exercise capacity (20). One study found no difference in outcomes in patients who met criteria for LVRS and underwent LVRS followed by lung transplant for ongoing symptoms, with the median time between LVRS and lung transplant being 33 months (21). Persistent or worsening dyspnea, increase in BODE or modified BODE score of more than 1 at 6 months post-LVRS are likely to have worse outcomes and could be considered candidates for lung transplantation (22). A decrease in score or less than one index increase was associated with lower mortality after LVRS. Likewise, bronchoscopic lung volume reduction with FDA approved valves could be considered as a bridge to transplant in the appropriately selected patients $(23,24)$. 
Table 5 Indications for lung transplant referral and listing in pulmonary vascular disease

Referral
NYHA class III-IV symptoms during escalating therapy
Rapidly progressive disease
Use of parenteral targeted PAH therapy
Known or suspected PVOD or pulmonary capillary
hemangiomatosis
Listing
NYHA class II-IV despite at least 3-month combination therapy
including prostanoids
Cl $<2$ L/min/m²
mRAP $>15$ mmHg
$6 M W D<350$ m
Significant hemoptysis, pericardial effusion, or progressive RHF

NYHA, New York Heart Association; PAH, pulmonary arterial hypertension; PVOD, pulmonary veno-occlusive disease; $\mathrm{Cl}$, cardiac index; mRAP, mean right atrial pressure; 6MWD, six-minute walk distance; RHF, right heart failure.

\section{Group B: pulmonary vascular disease}

The diseases included in this group B (1):

* Idiopathic or primary pulmonary arterial hypertension;

* Eisenmenger's syndrome;

* Chronic thromboembolic disease related pulmonary hypertension;

* Pulmonary veno-occlusive disease.

\section{IPAH}

The number of lung transplants for pulmonary arterial hypertension (PAH) has seen a decrease over the last two decades (9), and this is largely due to the improved survival with medical therapy for PAH $(25,26)$. Lung transplant is nowadays indicated for patients who show evidence of persistent deterioration despite aggressive and optimized medical treatment. Most centers perform just lung transplant alone in comparison to heart-lung transplant in the past for IPAH. The indications for lung transplant referral and listing in pulmonary vascular disease are summarized in Table 5.

Despite improvements in targeted medical therapy, PAH still has a relatively poor prognosis. The timing for referral in $\mathrm{PAH}$ patients remains a challenge, and the window of transplant can be narrow. In addition, patients with pulmonary hypertension have higher rates of perioperative complications, manifested by higher rates of primary graft dysfunction (PGD) and right ventricular failure $(9,27,28)$. There has been persistent evidence of an association between pulmonary arterial pressure (PAP), recipient BMI, female sex, and the rates of post-operative PGD $(27,29)$. The use of cardiopulmonary bypass has also been associated with increased risk for PGD. This poses yet another challenge since many patients who deteriorate rapidly require bridging strategies. There seems to be emerging evidence that an application of prolonged perioperative strategy of extracorporeal membrane oxygenation (ECMO) may be associated with improved outcomes $(30,31)$.

The Registry to Evaluate Early and Long-term PAH Disease Management (REVEAL) report identified and confirmed several risk factors associated with higher mortality. These are listed in Table 6 (32). Other factors associated with poor prognosis found in other studies are also listed in Table 6 (33-36). Heart rate recovery-1 (HRR1) is defined as the difference in heart rate at end of 6-minute walk test $(6 \mathrm{MWT})$ and 1 -minute post-resting after the test. A difference greater than 16 was found to carry a worse prognosis (37) (Table 6).

\section{Group C: infectious lung disease}

The diseases included in this group C (1):

* CF;

* Immune deficiency syndromes like IgG deficiency.

\section{CF}

Despite the significantly improved survival in CF over the last decades (38), many patients continue to have progressive disease and require lung transplant. Compared to other indications for lung transplant, the 5 -year lung transplant survival rates in CF are significantly better (9). This is largely due to the younger age of CF patients at the time of transplant. In addition, studies report improved quality of life among transplant recipients for CF $(39,40)$. The indications for lung transplant referral and listing are summarized in Table 7.

In patients who meet criteria for referral, a careful assessment should be made to determine their predicted survival and timing of transplant, which is not clearly demarcated. Several factors have been associated with increased mortality in CF patients, the most useful of which has been the $\mathrm{FEV}_{1}$ as a surrogate for disease progression and mortality. In earlier studies, an $\mathrm{FEV}_{1}<30 \%$ has been 
Table 6 Mortality risk factors in IPAH

Men $>60$ years of age

Evidence of right heart failure (elevated BNP, increased mean right atrial pressure and increased PVR, elevated troponin)

WHO functional class III-IV

Family history of IPAH

All-cause hospitalizations within the last 6 months

$\mathrm{PAH}$ associated with portal hypertension (PoPH) or scleroderma and CTD

Hyponatremia

Serum bilirubin level

Unchanged or increased serum bilirubin level despite medical

therapy

Renal insufficiency

Pericardial effusion

Resting systolic BP $<110 \mathrm{mmHg}$

Resting hear rate $>92$ beats per minutes

Six-minute walk distance (6MWD) $<166 \mathrm{~m}$

(6MWD $>440 \mathrm{~m}-$ better survival)

Low diffusing capacity

Heart rate recovery $<16$

IPAH, idiopathic pulmonary arterial hypertension; BNP, brain natriuretic peptide; PVR, pulmonary vascular resistance; WHO, World Health Organization; PAH, pulmonary arterial hypertension; PoPH, portopulmonary hypertension; CTD, connective tissue disease; BP, blood pressure.

associated with a 2-year mortality rate of approximately $50 \%(2,41)$, while later studies report the rate of decline in $\mathrm{FEV}_{1}$ to be a more reliable predictor of mortality in $\mathrm{CF}$ $(42,43)$. Other factors associated with increased mortality include female sex (41), shorter height (44), hypercapnia, pulmonary hypertension (45-47), pneumothorax (48), and shorter 6MWD (49) (see Table 8). In an attempt to come up with a mortality predictive model for CF, Mayer-Hamblett et al. incorporated several variables that were found to be associated mortality, including age, height, $\mathrm{FEV}_{1}$, respiratory microbiology, hospitalizations into a 2-year mortality predictive model. However, similar to $\mathrm{FEV}_{1}$, this was shown to have a low positive predictive value of $33 \%$ (50).

Infections in CF patients and their impact on lung transplantation

The most common bacteria found in the airways of CF patients are Staphylococcus and Pseudomonas. The resistance
Table 7 ISHLT indications for lung transplant referral and listing in cystic fibrosis

Referral

$\mathrm{FEV}_{1} \leq 30 \%$

Patient with advanced disease and rapidly falling $\mathrm{FEV}_{1}$ despite optimal treatment, especially female patients infected with NTM or BCC

$6 \mathrm{MWD}<400 \mathrm{~m}$

$\mathrm{PH}$ in absence of hypoxic exacerbation

Increased frequency of exacerbations with:

ARF requiring NIV

Increased antibiotic resistance and poor clinical recovery

Pneumothorax

Worsening nutritional status

Life threatening hemoptysis despite bronchial embolization

Listing

Chronic respiratory failure

With hypoxia $\mathrm{PaO}_{2}<60 \mathrm{mmHg}$

With hypercapnia $\mathrm{PaCO}_{2}>50 \mathrm{mmHg}$

Long-term NIV therapy

$\mathrm{PH}$

Frequent hospitalizations

Rapid lung function decline

WHO functional class IV

$\mathrm{FEV}_{1}$, forced expiratory volume in 1 second; NTM, non-tuberculous mycobacteria; BCC, Burkholderia cepacia complex; 6MWD, six-minute walk distance; $\mathrm{PH}$, pulmonary hypertension; ARF, acute respiratory failure; NIV, noninvasive ventilation; $\mathrm{PaO}_{2}$, partial pressure of oxygen; $\mathrm{PaCO}_{2}$, partial pressure of carbon dioxide; $\mathrm{PH}$, pulmonary hypertension; WHO, World Health Organization.

patterns of these organisms largely do not preclude patients from consideration for lung transplantation. All CF patients should be screened for non-tuberculous mycobacteria, Burkbolderia cepacia (B. cepacia) complex and fungal organisms. CF patients who are infected with $B$. cepacia complex have been shown to have a more rapid decline in $\mathrm{FEV}_{1}$, higher pre-transplant and even post-transplant mortality (51-55). Notably the species Burkbolderia cenocepacia (B. cenocepacia) has been a problematic infection both pre- and post-lung transplant. While there are few centers who have transplanted patients with $B$. cenocepacia, the overall survival in this cohort is lower than the other 
Table 8 Mortality risk factors in cystic fibrosis

Low FEV $_{1}$
Female sex
Shorter height
Hypercapnia
Pulmonary hypertension
Pneumothorax
Shorter 6MWD
Burkholderia cepacia infection

$\mathrm{FEV}_{1}$, forced expiratory volume in 1 second; 6MWD, six-minute walk distance.

Table 9 Indications for lung transplant referral and listing in ILD

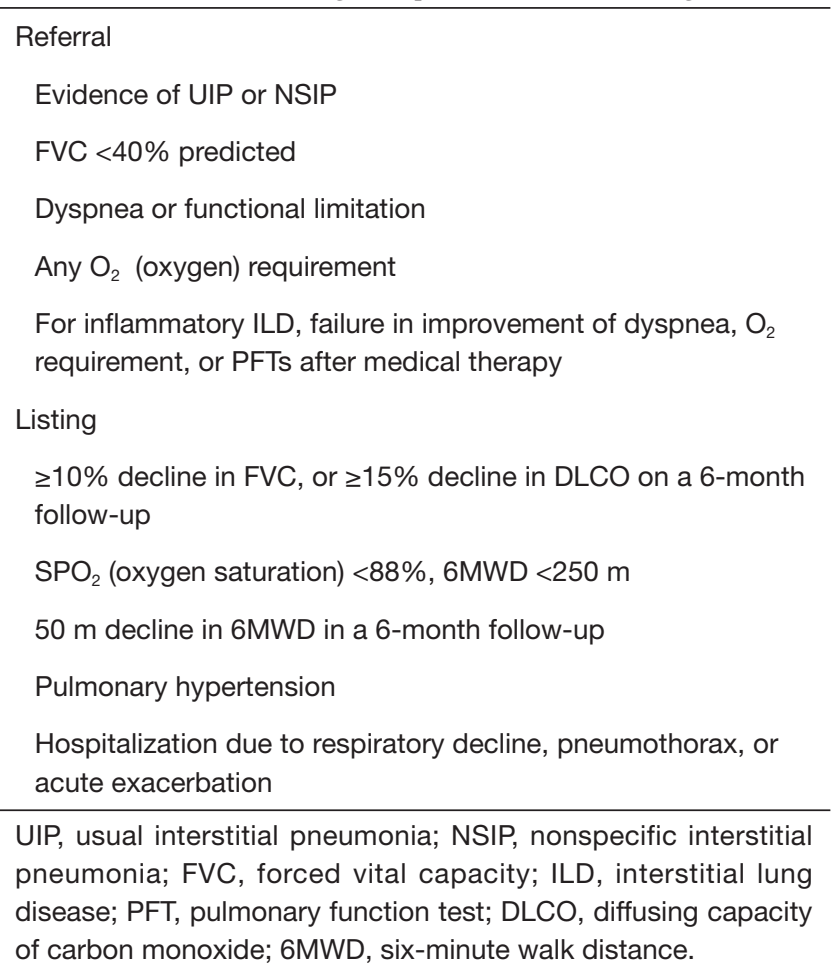

CF patients at the same center (56). Most centers do not consider accepting CF patients with B. cenocepacia infection. The current ISHLT guidelines recommend that all patients referred for lung transplant should be tested for B. cepacia. While infection with $B$. cenocepacia does not constitute an absolute contraindication, the ISHLT recommends that centers accepting these cases to have the resources necessary to test methods for controlling and preventing recurrent disease (2).
Non-tuberculosis mycobacteria are found in approximately $10-20 \%$ of the CF patients in sputum cultures (57). The predominant species include Mycobacterium avium complex and Mycobacterium abscessus. Disseminated and untreated infections in CF patients make them ineligible for transplant in many centers. Mycobacterium abscessus can be problematic with persistent, recurrent and difficult to eradicate soft tissue and mediastinal infections despite optimal surgical and medical treatment. Many centers do not consider transplant in patients with Mycobacterium abscessus infection but it is not an absolute contraindication and there are centers that have transplanted with acceptable or comparable outcomes (58). Outcomes are considered to be more favorable in patients with non-disseminated infections who demonstrate a favorable susceptibility pattern and an improvement with treatment. Monthly respiratory cultures are needed for monitoring response to therapy.

\section{Group D: restrictive lung disease}

The diseases included in this group D (1):

* IPF;

* Eosinophilic granulomatosis;

* Sarcoidosis with mean pulmonary artery pressure $\geq 30 \mathrm{mmHg}$;

* Scleroderma/CREST syndrome;

* Bronchoalveolar carcinoma;

* Bronchiolitis obliterans syndrome (BOS) following lung transplant;

* Primary graft failure following lung transplant.

\section{IPF}

IPF is a rapidly progressive disease with a median survival of 2-3 years from the time of diagnosis and a 5 -year survival of about $25 \%(59,60)$. IPF is the most common subtype of ILD and has been associated with worse outcomes when compared to other forms of ILD (61). Other ILD that may carry a similar course as IPF include fibrotic non-specific interstitial pneumonia (NSIP), progressive ILDs refractory to immunomodulation therapy. The current lung transplant indications for referral and listing in ILDs are summarized in Table 9.

The high mortality in IPF, along with the implementation of the LAS has been responsible for the dramatic increase in the number of lung transplant recipients with IPF over the last two decades, and warrants earlier referral for lung transplant evaluation (4). Despite FDA approval of the anti-fibrotic agents nintedanib and 
Table 10 Risk factors for mortality in IPF

Low baseline $\mathrm{SpO}_{2}$
Increase in desaturation during six-minute walk test
FVC decline $>10 \%$ in 6 months
Decrease in $\mathrm{DLCO}>15 \%$ in 6 months
Decrease in $6 \mathrm{MWD}>200$ feet in 6 months
Elevated PAP
dyspnea severity
Higher Gender-Age-Physiology (GAP) stage
SpO ${ }_{2}$, oxygen saturation; $6 \mathrm{MWD}$, six-minute walk distance; FVC,
forced vital capacity; PAP, pulmonary arterial pressure; DLCO,
diffusing capacity of carbon monoxide.

pirfenidone, no medical therapy has been shown to have a clearly established impact on mortality (62). However, few recent studies have shown improved overall survival with these drugs $(63,64)$. With the availability and use of these drugs, it may be possible to extend the "transplant window" in certain subgroups of this cohort.

Relative to COPD, mortality predictors in IPF have been well studied, and a variety of mortality prediction models that include age, sex, BMI, forced vital capacity (FVC), $\mathrm{FEV}_{1} / \mathrm{FVC}$ ratio, diffusing capacity of carbon monoxide (DLCO), 6MWD, dyspnea severity, and Gender-AgePhysiology (GAP) stage, have been established (65-70) (see Table 10). However, these variables do not reliably predict the risk of disease progression. Recent evidence highlights a stepwise rather than linear progression in IPF, with several studies showing that changes in FVC, dyspnea scores and $6 \mathrm{MWT}$ are not necessarily associated with changes in the future (71-73). This may have important future implications on selection of patients with IPF for lung transplant. Given the high unpredictability of IPF progression, there has been interest in recent years in identifying biomarkers that could predict disease progression. In a study by Prasse et al., CCL18, a biomarker that was shown abundant production in alveolar macrophages, was found to correlate to pulmonary fibrotic activity, disease progression, and mortality $(74,75)$. However, in a recent prospective, casecontrolled multicenter study by Raghu et al., CCL18 was associated with IPF but did not predict disease progression (76). As of now, there are no biomarkers that have been conclusively shown to have a correlation with disease progression or mortality. A clinical risk-scoring model using age, respiratory hospitalizations, and percent predicted FVC, and a 24-week change in FVC seemed to produce 1-year mortality results consistent with observed data (43).

Connective tissue disease related ILD (CTD-ILD) are usually more slowly progressive and more responsive to immunomodulatory therapies. It is important to consider the potential side effects of therapies especially if being considered in patients with severe lung disease and are or approaching the transplant window. Survival after transplant in CTD-ILD patients was similar to patients who lung transplant for IPAH or IPF irrespective of the type of ILD (9).

\section{Multi organ transplant}

Most of the patients who require multi-organ transplant usually fall in to one of the following two categories: (I) patients with more than one end stage organ dysfunctions who meet criteria for each organ transplantation independently or, (II) if a post-transplant organ (nontransplanted organ) dysfunction would be anticipated if the patient were to receive either single organ alone. Candidates for multi-organ transplants are usually less than 60 years of age.

\section{Heart-lung transplantation}

Currently, there are very few centers doing heart-lung transplants and there are less than a hundred heart-lung transplants done per year worldwide. Previously, the survival of heart-lung transplant was inferior to bilateral lung transplant only, but in the most recent cohort, it was at least similar and patients who survived the first year had better long-term survival (9). The most common indication of heart lung transplant in the past was IPAH but in the last decade, complex congenital heart disease with Eisenmenger's syndrome is a more common indication. Other indications for heart-lung transplant include end stage lung disease with decreased left ventricular ejection fraction combined with low cardiac index, fibrosis or infarction on the right ventricle with failure seen on imaging, sarcoidosis with involvement of both the heart and lung to a significant degree and finally for patients with cardiomyopathy who have a residual increased pulmonary vascular resistance despite adequate medical or surgical treatment (typically assist device) (77). End stage lung disease with patients with cardiac lesions like valvular abnormalities, coronary lesions, repairable congenital 
defects usually are candidates for bilateral lung transplant with corrective surgery provided adequate cardiac function is present and expected to continue unaffected (78). In addition, time to bilateral lung transplant are shorter compared to heart-lung transplant. This is secondary to allocation policies in the United States (7)

\section{Lung and abdominal organ transplant}

Survival outcomes following combined organ transplant are better than lung transplant alone but are worse than abdominal organ survival independently (79). The most common combined organ transplant is usually lung and kidney but in most scenarios, it is not performed simultaneously (most commonly the kidney transplant is done following lung transplant). These are largely cases of end stage kidney failure secondary to chronic use of immunosuppressive medications (primarily calcineurin inhibitors). Kidney dysfunction following lung transplant is one of the most significant risk factors for poor survival outcomes.

Lung-liver transplants are mostly done for the same disease process such as $\mathrm{CF}$ or alpha-1 antitrypsin deficiency affecting both the lung and liver. However, it is also done in end stage lung disease patients with biopsy proven cirrhosis and high portal gradient in carefully selected patients.

\section{Conclusions}

Lung transplantation continues to be a crucial treatment modality for end stage lung disease. Over the last decades, significant improvements have been made to the process of lung procurement and allocation, preservation, surgical techniques and medical advancement resulting in significant decrease in waitlist mortality, improved outcomes, and increase in the number of transplant procedures. Despite these developments, the current guidelines for lung transplant referral and listing remain largely dependent on expert opinion. There is a continued trend toward adopting a more personalized approach that emphasizes the unique individual's disease-specific survival predictors when evaluating lung transplant candidates that will result in further advancements in the field of lung transplant.

\section{Acknowledgments}

None.

\section{Footnote}

Conflicts of Interest: The authors have no conflicts of interest to declare.

\section{References}

1. Egan TM, Murray S, Bustami RT, et al. Development of the new lung allocation system in the United States. Am J Transplant 2006;6:1212-27.

2. Weill D, Benden C, Corris PA, et al. A consensus document for the selection of lung transplant candidates: 2014--an update from the Pulmonary Transplantation Council of the International Society for Heart and Lung Transplantation. J Heart Lung Transplant 2015;34:1-15.

3. Orens JB, Estenne M, Arcasoy S, et al. International guidelines for the selection of lung transplant candidates: 2006 update--a consensus report from the Pulmonary Scientific Council of the International Society for Heart and Lung Transplantation. J Heart Lung Transplant 2006;25:745-55.

4. International guidelines for the selection of lung transplant candidates. The American Society for Transplant Physicians (ASTP)/American Thoracic Society(ATS)/ European Respiratory Society(ERS)/International Society for Heart and Lung Transplantation(ISHLT). Am J Respir Crit Care Med 1998;158:335-9.

5. Egan TM, Bennett LE, Garrity ER, et al. Predictors of death on the UNOS lung transplant waiting list: results of a multivariate analysis. J Heart Lung Transplant 2001;20:242.

6. Organ Procurement and Transplantation Network. Health Resources and Services Administration, HHS. Final rule. Fed Regist 1999;64:56650-61.

7. Colvin-Adams $M$, Valapour $M$, Hertz $M$, et al. Lung and heart allocation in the United States. Am J Transplant 2012;12:3213-34.

8. Egan TM, Edwards LB. Effect of the lung allocation score on lung transplantation in the United States. J Heart Lung Transplant 2016;35:433-9.

9. Yusen RD, Edwards LB, Dipchand AI, et al. The Registry of the International Society for Heart and Lung Transplantation: Thirty-third Adult Lung and HeartLung Transplant Report-2016; Focus Theme: Primary Diagnostic Indications for Transplant. J Heart Lung Transplant 2016;35:1170-84.

10. Lederer DJ, Wilt JS, D'Ovidio F, et al. Obesity and underweight are associated with an increased risk of death 
after lung transplantation. Am J Respir Crit Care Med 2009;180:887-95.

11. Shigemura N, Bhama J, Gries CJ, et al. Lung transplantation in patients with prior cardiothoracic surgical procedures. Am J Transplant 2012;12:1249-55.

12. Fletcher $\mathrm{C}$, Peto R. The natural history of chronic airflow obstruction. Br Med J 1977;1:1645-8.

13. Celli BR. Predictors of mortality in COPD. Respir Med 2010;104:773-9.

14. Soler-Cataluña JJ, Martinez-Garcia MA, Sanchez LS, et al. Severe exacerbations and BODE index: two independent risk factors for death in male COPD patients. Respir Med 2009; 103:692-9.

15. Martinez FJ, Foster G, Curtis JL, et al. Predictors of mortality in patients with emphysema and severe airflow obstruction. Am J Respir Crit Care Med 2006;173:1326-34.

16. Oga T, Nishimura K, Tsukino M, et al. Analysis of the factors related to mortality in chronic obstructive pulmonary disease: role of exercise capacity and health status. Am J Respir Crit Care Med 2003;167:544-9.

17. Yoshimura K, Maekura R, Hiraga T, et al. Identification of three exercise-induced mortality risk factors in patients with COPD. COPD 2014;11:615-26.

18. Soler-Cataluña JJ, Martinez-Garcia MA, Roman Sanchez P, et al. Severe acute exacerbations and mortality in patients with chronic obstructive pulmonary disease. Thorax 2005;60:925-31.

19. Connors AF Jr, Dawson NV, Thomas C, et al. Outcomes following acute exacerbation of severe chronic obstructive lung disease. The SUPPORT investigators (Study to Understand Prognoses and Preferences for Outcomes and Risks of Treatments). Am J Respir Crit Care Med 1996;154:959-67.

20. Fishman A, Martinez F, Naunheim K, et al. A randomized trial comparing lung-volume-reduction surgery with medical therapy for severe emphysema. N Engl J Med 2003;348:2059-73.

21. Tutic M, Lardinois D, Imfeld S, et al. Lung-volume reduction surgery as an alternative or bridging procedure to lung transplantation. Ann Thorac Surg 2006;82:208-13; discussion 213.

22. Imfeld S, Bloch KE, Weder W, et al. The BODE index after lung volume reduction surgery correlates with survival. Chest 2006;129:873-8.

23. Valipour A. Valve therapy in patients with emphysematous type of chronic obstructive pulmonary disease (COPD): from randomized trials to patient selection in clinical practice. J Thorac Dis 2018;10:S2780-96.
24. Criner GJ, Sue R, Wright S, et al. A Multicenter Randomized Controlled Trial of Zephyr Endobronchial Valve Treatment in Heterogeneous Emphysema (LIBERATE). Am J Respir Crit Care Med 2018;198:1151-64.

25. Conte JV, Gaine SP, Orens JB, et al. The influence of continuous intravenous prostacyclin therapy for primary pulmonary hypertension on the timing and outcome of transplantation. J Heart Lung Transplant 1998;17:679-85.

26. Sitbon O, Humbert M, Nunes H, et al. Long-term intravenous epoprostenol infusion in primary pulmonary hypertension: prognostic factors and survival. J Am Coll Cardiol 2002;40:780-8.

27. Porteous MK, Lee JC, Lederer DJ, et al. Clinical Risk Factors and Prognostic Model for Primary Graft Dysfunction after Lung Transplantation in Patients with Pulmonary Hypertension. Ann Am Thorac Soc 2017;14:1514-22.

28. Singer JP. Pulmonary Hypertension and Primary Graft Dysfunction in Lung Transplant Recipients: We Still Have a Long Way to Go. Ann Am Thorac Soc 2017;14:1504-5.

29. Liu Y, Su L, Jiang SJ. Recipient-related clinical risk factors for primary graft dysfunction after lung transplantation: a systematic review and meta-analysis. PLoS One 2014;9:e92773.

30. Salman J, Ius F, Sommer W, et al. Mid-term results of bilateral lung transplant with postoperatively extended intraoperative extracorporeal membrane oxygenation for severe pulmonary hypertension. Eur J Cardiothorac Surg 2017;52:163-70.

31. Moser B, Jaksch P, Taghavi S, et al. Lung transplantation for idiopathic pulmonary arterial hypertension on intraoperative and postoperatively prolonged extracorporeal membrane oxygenation provides optimally controlled reperfusion and excellent outcome. Eur J Cardiothorac Surg 2018;53:178-85.

32. Benza RL, Miller DP, Gomberg-Maitland M, et al. Predicting survival in pulmonary arterial hypertension: insights from the Registry to Evaluate Early and LongTerm Pulmonary Arterial Hypertension Disease Management (REVEAL). Circulation 2010;122:164-72.

33. Galiè N, Humbert M, Vachiery JL, et al. 2015 ESC/ERS Guidelines for the diagnosis and treatment of pulmonary hypertension: The Joint Task Force for the Diagnosis and Treatment of Pulmonary Hypertension of the European Society of Cardiology (ESC) and the European Respiratory Society (ERS): Endorsed by: Association for European Paediatric and Congenital Cardiology (AEPC), 
International Society for Heart and Lung Transplantation (ISHLT). Eur Heart J 2016;37:67-119.

34. Raina A, Humbert M. Risk assessment in pulmonary arterial hypertension. Eur Respir Rev 2016;25:390-8.

35. Takeda Y, Tomimoto S, Tani T, et al. Bilirubin as a prognostic marker in patients with pulmonary arterial hypertension. BMC Pulm Med 2010;10:22.

36. Xu XQ, Lv ZC, Liu QQ, et al. Direct bilirubin: A new risk factor of adverse outcome in idiopathic pulmonary arterial hypertension. Int J Cardiol 2017;228:895-9.

37. Minai OA, Nguyen Q, Mummadi S, et al. Heart rate recovery is an important predictor of outcomes in patients with connective tissue disease-associated pulmonary hypertension. Pulm Circ 2015;5:565-76.

38. Keogh RH, Szczesniak R, Taylor-Robinson D, et al. Upto-date and projected estimates of survival for people with cystic fibrosis using baseline characteristics: A longitudinal study using UK patient registry data. J Cyst Fibros 2018;17:218-27.

39. Vermeulen KM, van der Bij W, Erasmus ME, et al. Improved quality of life after lung transplantation in individuals with cystic fibrosis. Pediatr Pulmonol 2004;37:419-26.

40. Lynch JP 3rd, Sayah DM, Belperio JA, et al. Lung transplantation for cystic fibrosis: results, indications, complications, and controversies. Semin Respir Crit Care Med 2015;36:299-320.

41. Kerem E, Reisman J, Corey M, et al. Prediction of mortality in patients with cystic fibrosis. N Engl J Med 1992;326:1187-91.

42. Milla CE, Warwick WJ. Risk of death in cystic fibrosis patients with severely compromised lung function. Chest 1998;113:1230-4.

43. Augarten A, Akons H, Aviram M, et al. Prediction of mortality and timing of referral for lung transplantation in cystic fibrosis patients. Pediatr Transplant 2001;5:339-42.

44. Belkin RA, Henig NR, Singer LG, et al. Risk factors for death of patients with cystic fibrosis awaiting lung transplantation. Am J Respir Crit Care Med 2006;173:659-66.

45. Venuta F, Rendina EA, De Giacomo T, et al. Timing and priorities for cystic fibrosis patients candidates to lung transplantation. Eur J Pediatr Surg 1998;8:274-7.

46. Venuta F, Rendina EA, Rocca GD, et al. Pulmonary hemodynamics contribute to indicate priority for lung transplantation in patients with cystic fibrosis. J Thorac Cardiovasc Surg 2000;119:682-9.

47. Hayes D Jr, Tobias JD, Mansour HM, et al. Pulmonary hypertension in cystic fibrosis with advanced lung disease. Am J Respir Crit Care Med 2014;190:898-905.

48. Flume PA, Mogayzel PJ Jr, Robinson KA, et al. Cystic fibrosis pulmonary guidelines: pulmonary complications: hemoptysis and pneumothorax. Am J Respir Crit Care Med 2010;182:298-306.

49. Tuppin MP, Paratz JD, Chang AT, et al. Predictive utility of the 6-minute walk distance on survival in patients awaiting lung transplantation. J Heart Lung Transplant 2008;27:729-34.

50. Mayer-Hamblett N, Rosenfeld M, Emerson J, et al. Developing cystic fibrosis lung transplant referral criteria using predictors of 2-year mortality. Am J Respir Crit Care Med 2002;166:1550-5.

51. Alexander BD, Petzold EW, Reller LB, et al. Survival after lung transplantation of cystic fibrosis patients infected with Burkholderia cepacia complex. Am J Transplant 2008;8:1025-30.

52. Chaparro C, Maurer J, Gutierrez C, et al. Infection with Burkholderia cepacia in cystic fibrosis: outcome following lung transplantation. Am J Respir Crit Care Med 2001;163:43-8.

53. Jones AM, Dodd ME, Govan JR, et al. Burkholderia cenocepacia and Burkholderia multivorans: influence on survival in cystic fibrosis. Thorax 2004;59:948-51.

54. Frangolias DD, Mahenthiralingam E, Rae S, et al. Burkholderia cepacia in cystic fibrosis. Variable disease course. Am J Respir Crit Care Med 1999;160:1572-7.

55. Manno G, Dalmastri C, Tabacchioni S, et al. Epidemiology and clinical course of Burkholderia cepacia complex infections, particularly those caused by different Burkholderia cenocepacia strains, among patients attending an Italian Cystic Fibrosis Center. J Clin Microbiol 2004;42:1491-7.

56. Nash EF, Coonar A, Kremer R, et al. Survival of Burkholderia cepacia sepsis following lung transplantation in recipients with cystic fibrosis. Transpl Infect Dis 2010;12:551-4.

57. Adjemian J, Olivier KN, Prevots DR. Epidemiology of Pulmonary Nontuberculous Mycobacterial Sputum Positivity in Patients with Cystic Fibrosis in the United States, 2010-2014. Ann Am Thorac Soc 2018;15:817-26.

58. Lobo LJ, Chang LC, Esther CR Jr, et al. Lung transplant outcomes in cystic fibrosis patients with pre-operative Mycobacterium abscessus respiratory infections. Clin Transplant 2013;27:523-9.

59. Bjoraker JA, Ryu JH, Edwin MK, et al. Prognostic significance of histopathologic subsets in idiopathic 
pulmonary fibrosis. Am J Respir Crit Care Med 1998;157:199-203.

60. Raghu G, Collard HR, Egan JJ, et al. An official ATS/ ERS/JRS/ALAT statement: idiopathic pulmonary fibrosis: evidence-based guidelines for diagnosis and management. Am J Respir Crit Care Med 2011;183:788-824.

61. Thomeer MJ, Vansteenkiste J, Verbeken EK, et al. Interstitial lung diseases: characteristics at diagnosis and mortality risk assessment. Respir Med 2004;98:567-73.

62. King TE Jr, Bradford WZ, Castro-Bernardini S, et al. A phase 3 trial of pirfenidone in patients with idiopathic pulmonary fibrosis. N Engl J Med 2014;370:2083-92.

63. Harari S, Caminati A, Poletti V, et al. A RealLife Multicenter National Study on Nintedanib in Severe Idiopathic Pulmonary Fibrosis. Respiration 2018;95:433-40.

64. Margaritopoulos GA, Trachalaki A, Wells AU, et al. Pirfenidone improves survival in IPF: results from a reallife study. BMC Pulm Med 2018;18:177.

65. Ley B, Collard HR, King TE Jr. Clinical course and prediction of survival in idiopathic pulmonary fibrosis. Am J Respir Crit Care Med 2011;183:431-40.

66. Ley B, Ryerson CJ, Vittinghoff E, et al. A multidimensional index and staging system for idiopathic pulmonary fibrosis. Ann Intern Med 2012;156:684-91.

67. du Bois RM, Weycker D, Albera C, et al. Ascertainment of individual risk of mortality for patients with idiopathic pulmonary fibrosis. Am J Respir Crit Care Med 2011;184:459-66.

68. Brown AW, Shlobin OA, Weir N, et al. Dynamic patient counseling: a novel concept in idiopathic pulmonary fibrosis. Chest 2012;142:1005-10.

69. Flaherty KR, Andrei AC, Murray S, et al. Idiopathic pulmonary fibrosis: prognostic value of changes in physiology and six-minute-walk test. Am J Respir Crit Care Med 2006;174:803-9.

70. Collard HR, King TE Jr, Bartelson BB, et al. Changes in clinical and physiologic variables predict survival in

Cite this article as: Shweish O, Dronavalli G. Indications for lung transplant referral and listing. J Thorac Dis 2019;11(Suppl 14):S1708-S1720. doi: 10.21037/jtd.2019.05.09 idiopathic pulmonary fibrosis. Am J Respir Crit Care Med 2003;168:538-42.

71. Salisbury ML, Xia M, Zhou Y, et al. Idiopathic Pulmonary Fibrosis: Gender-Age-Physiology Index Stage for Predicting Future Lung Function Decline. Chest 2016;149:491-8.

72. Schmidt SL, Tayob N, Han MK, et al. Predicting pulmonary fibrosis disease course from past trends in pulmonary function. Chest 2014;145:579-85.

73. Ley B, Bradford WZ, Vittinghoff E, et al. Predictors of Mortality Poorly Predict Common Measures of Disease Progression in Idiopathic Pulmonary Fibrosis. Am J Respir Crit Care Med 2016;194:711-8.

74. Prasse A, Probst C, Bargagli E, et al. Serum CCchemokine ligand 18 concentration predicts outcome in idiopathic pulmonary fibrosis. Am J Respir Crit Care Med 2009;179:717-23.

75. Prasse A, Pechkovsky DV, Toews GB, et al. CCL18 as an indicator of pulmonary fibrotic activity in idiopathic interstitial pneumonias and systemic sclerosis. Arthritis Rheum 2007;56:1685-93.

76. Raghu G, Richeldi L, Jagerschmidt A, et al. Idiopathic Pulmonary Fibrosis: Prospective, Case-Controlled Study of Natural History and Circulating Biomarkers. Chest 2018;154:1359-70.

77. Mehra MR, Canter CE, Hannan MM, et al. The 2016 International Society for Heart Lung Transplantation listing criteria for heart transplantation: A 10-year update. J Heart Lung Transplant 2016;35:1-23.

78. Choong CK, Sweet SC, Guthrie TJ, et al. Repair of congenital heart lesions combined with lung transplantation for the treatment of severe pulmonary hypertension: a 13-year experience. J Thorac Cardiovasc Surg 2005;129:661-9.

79. Wolf JH, Sulewski ME, Cassuto JR, et al. Simultaneous thoracic and abdominal transplantation: can we justify two organs for one recipient? Am J Transplant 2013;13:1806-16. 